\title{
LAS VISITAS GUIADAS EN EL JARDÍN BOTÁNICO DE MATANZAS, CUBA
}

\section{GUIDED VISITS IN THE BOTANICAL GARDEN OF MATANZAS, CUBA}

\author{
Ainel González Robledo ${ }^{1}$ \\ Lenia Robledo Ortega ${ }^{2}$ \\ Amalia Enríquez Rodríguez ${ }^{3}$ \\ Yamilé Rodríguez Bárzaga ${ }^{4}$
}

Página | 152

\section{RESUMEN}

Los jardines botánicos juegan un papel muy importante para la conservación de las especies de plantas en peligro de desaparecer de la naturaleza, de la flora local, nacional y en educación ambiental. El Jardín Botánico de Matanzas tiene experiencia de más de quince años, con actividades de educación ambiental, donde los estudiantes de los diversos niveles de enseñanza son los principales actores. Las visitas guiadas y otras actividades que se realizan en el jardín, se adecuan a las características de los diferentes grupos y elevan los conocimientos acerca de las plantas y la importancia de su protección. Los resultados de las visitas guiadas avalan el trabajo de la educación ambiental en el jardín botánico de Matanzas, Cuba.

Palavras chave: Jardín botânico; Medioambiente; Visita guiada.

\begin{abstract}
The Botanical Garden play a paper very important for the conservation of the species in danger of disappear of the nature, the local flora, national and the environmental education. The Botanical Garden of Matanzas had experience of more than 15 years, making activities of Environmental education. The students of the different levels of education are the principal participants in the guide visit to the garden, making a group of activities who make possible increase the knowledge about the plants and the importance of their protection. The results of the guide visits show the work of the environmental education in the Botanical Garden of Matanzas, Cuba.
\end{abstract}

Keywords: Botanical garden; Environmental; Guide visit.

\footnotetext{
${ }^{1}$ Profesor Auxiliar, MSc. En Botánica. Universidad de Matanzas, Cuba. aingrobledo@gmail.com

${ }^{2}$ Profesora Auxiliar. MSc. En Botánica Universidad de Matanzas, Cuba. lenia.robledo@umcc.cu

${ }^{3}$ Profesora Auxiliar. MSc. En Botánica Universidad de Matanzas, Cuba. amaliaher48@yahoo.com

${ }^{4}$ Profesora Instructora. MSc. En Agropecuaria. Universidad de Matanzas, Cuba.

yamile.rodriguez@umcc.cu
} 


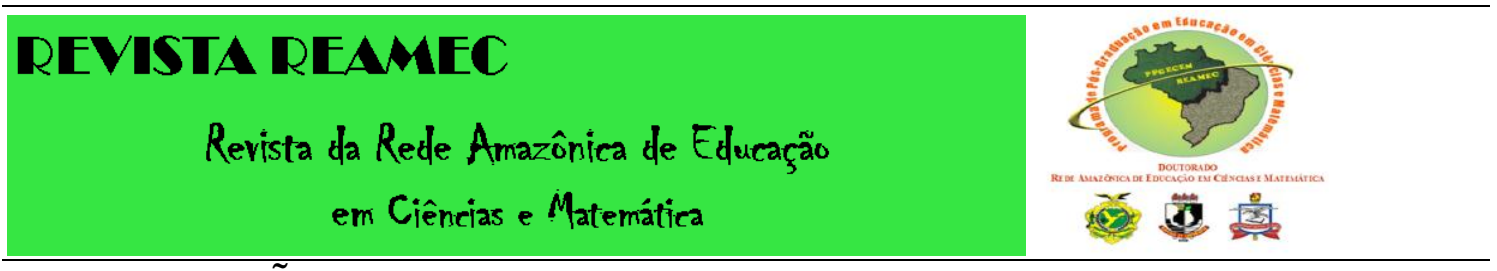

1 INTRODUÇÃ̃O

Los jardines botánicos son reconocidos como legados insustituibles para la investigación de las plantas, de sus usos en beneficios de la humanidad; para educar al pueblo sobre los aspectos de la vida de las plantas, su diversidad y su interdependencia con otros elementos del ecosistema, como eficaces instrumentos para la conservación de la flora y el desarrollo de una cultura ambiental (LINARES, 1994; RANGEL, 1994).

La educación ambiental está unida al rol de los Jardines Botánicos, ya que los mismos tienen las potencialidades para incidir en la visión de un enfoque ambiental y en el comportamiento de las personas que por razones diversas visitan sus instalaciones. Es un proceso formativo, integral e interdisciplinario que considera el ambiente como un todo y busca involucrar a la población en general a la solución de conflictos ambientales, habilidades y la toma de decisiones (GALLEGO, 2010).

A partir de la educación ambiental se propicia adquisición de una conciencia para la conservación del medio ambiente mediante la generación de alternativas de los recursos naturales y el mejoramiento del bienestar humano. Los jardines botánicos como instituciones científicas vinculadas al medio ambiente desarrollan acciones que influyen en la disminución de los impactos ambientales sobre los ecosistemas y proveen conocimientos que son partes inseparables de la conciencia ciudadana contemporánea y conservación de los valores forestales patrimoniales (ÁLVAREZ, 2013).

El objetivo de este trabajo es exponer las experiencias del Jardín Botánico de Matanzas en el trabajo de la educación ambiental a partir de las visitas guiadas.

\section{DESENVOLVIMENTO}

La educación ambiental al ser un proceso continuo que abarca a toda la sociedad, tiene diversos escenarios que se diferencian por la dinámica y naturaleza de los procesos educativos, los actores y públicos metas involucrados, la forma y medios de aprendizaje, así como el grado de participación.

Entre los escenarios para el desarrollo de la educación ambiental se encuentran todas las escuelas y universidades donde se desarrollan procesos educativos establecidos por programas oficiales desde edades tempranas y conducidos por profesionales de educación. También participan instituciones científico-recreativas como acuarios, 


\section{REVISTA REAMEC}

Revista da Rede Amazônica de Educação

em Ciências e Matemática

$\overline{\text { jardines botánicos, museos, zoológicos, así como las áreas protegidas y las comunidades }}$ poblacionales humanas.

Dentro de las actividades de educación ambiental realizadas en el Jardín Botánico de Matanzas, se encuentran las visitas guiadas, esta actividad tiene como objetivo: Realizar recorridos por las colecciones de plantas en las áreas del jardín, observar la unidad y diversidad de la flora, establecer las características que permiten reunir en diversas familias de plantas, a los ejemplares que son parte de la colección.

Durante las visitas guiadas se deben tener en cuenta los siguientes aspectos (ROBLEDO, 2015):

Que la longitud del recorrido y áreas del jardín a mostrar esté en correspondencia con el nivel educacional y las edades de los participantes.

Que la profundidad de las explicaciones se adecuen a las edades y el nivel de los escolares o solicitudes de los organismos y otras entidades.

Se deben mostrar las áreas en su totalidad y concluir teniendo en cuenta los valores de especies seleccionadas, con su origen y estatus de conservación.

Reforzar con hechos históricos el recorrido y la memoria de científicos notables cubanos, extranjeros y el personal que atiende las colecciones del jardín.

Entre los resultados priorizados en las visitas guiadas en el Jardín Botánico de Matanzas, está el incentivar en los estudiantes, la necesidad de proteger las especies vegetales y la conservación de las que presentan mayor riesgo de desaparecer en la naturaleza.

La representación de las características ecológicas en las diferentes áreas permite transmitir a los estudiantes conocimientos acerca de las adaptaciones de las plantas. Ejemplo: En el área de cactáceas y otras suculentas, pueden apreciar las características de esas familias y sus adaptaciones a medioambientes con escasas precipitaciones. Las plantas desarrollan largas raíces, así como sus hojas están transformadas en espinas que regulan la evaporación del agua.

Los jardines botánicos lugares con gran experiencia en el manejo de semillas y con personal capacitado para el cultivo de plantas se convierten en los centros apropiados para desarrollar estrategias de conservación ex-situ de especies amenazadas (BACCHETA et al., 2008). 


\section{REVISTA REAMEC}

Revista da Rede Amazônica de Educação

em Ciências e Matemática

A partir de la incorporación del jardín botánico de matanzas en la red de jardines botánicos de la República de Cuba en el año 2002, se dio prioridad en el jardín al establecimiento de las colecciones ex-situ comprometidas con la red de jardines, en especial con endemismos catalogados en peligro crítico de extinción (ROBLEDO et al., 2009; ROBLEDO et al., 2009-2010; ROBLEDO et al., 2015).

\subsection{Visitas al JBM y actividades de educación ambiental}

El jardín se encuentra en el campus universitario de la Universidad de Matanzas, esto significa que el objeto social es diferente al de otros jardines de la Red Nacional, por lo que las visitas al jardín están limitadas a estudiantes de los diversos niveles de enseñanzas (GONZÁLEZ et al., 2014).

La zonificación de las áreas didácticas del jardín son: sub-área (1), Coníferas; (2), Relación Planta-ambiente y (3), Bosque Martiano; así como tiene un Área de Colecciones de conservación y vivero. Estas áreas son utilizadas para los recorridos con los visitantes a partir de las solicitudes de los diversos centros educacionales y medioambientalistas (GONZÁLEZ et al., 2014).

Entre los centros educacionales atendidos están: círculos infantiles, escuelas de enseñanza primaria, escuelas de secundaria básica, escuelas de enseñanza media superior, enseñanza superior y otras instituciones como círculos de abuelos.

Las acciones de educación ambiental en el jardín fueron elaboradas a partir de las características de las áreas y los diferentes grupos estudiantiles. Entre las actividades diseñadas, según González (2001), están:

1. Visita guiada

2. ¿Dónde viven las plantas?

3. Siembra de cactus

4. Trabajo con Clave

5. Juego de "La fotosíntesis"

6. Diferentes usos de las plantas

7. Naturaleza y literatura

8. Pintura y naturaleza

Se confeccionó un folleto con estas actividades, cada una con los objetivos, orientaciones generales, particularidades según los niveles educacionales. Se muestra una como ejemplo. 


\section{REVISTA REAMEC}

Revista da Rede Amazônica de Educação

em Ciências e Matemática

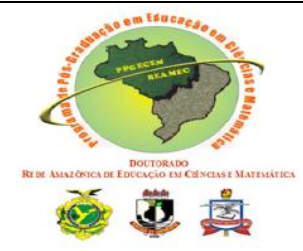

\subsection{Encuentro \# 1.- Visita guiada}

En este encuentro se les explica a los estudiantes los objetivos del jardín botánico y algunos aspectos históricos. Posteriormente se les explica las características principales de las especies de las familias representadas en el área didáctica, destacando las diferencias que existen entre ellas en cuanto a su forma, tamaño, color y las semejanzas relacionadas con las funciones como organismo vivo.

\section{Actividades}

1- Explicación de datos generales e históricos relacionados con el jardín

2- Observación gráfica en fotos o computarizadas de aspectos generales sobre el jardín.

3- Recorrido por las áreas didácticas (sub- área 1, 2 y 3) para la observación de las colecciones de plantas por familias.

A través de preguntas debe lograrse que los alumnos:

-Expliquen las semejanzas y diferencias de las plantas a partir de la observación durante el recorrido.

-Expliquen las diferencias en los mecanismos de reproducción entre los helechos $\mathrm{y}$ las angiospermas

-Valoren el cuidado del medio ambiente a partir de la importancia de las plantas para el resto de los organismos.

\section{Nivel Primario}

Para los estudiantes de nivel primario, las observaciones serán generales, las explicaciones breves, buscando que se logren los objetivos de la actividad de manera secuencial, recordando que en sus programas educacionales, éstos se tratan para explicar la interacción entre el hombre y la naturaleza.

\section{Nivel Secundario y Pre-Universitario}

Para los estudiantes de nivel secundario y de pre-universitario se precisarán elementos acerca de la anatomía y fisiología de las plantas, grupos y diversidad entre musgos, helechos, coníferas y angiospermas; así como su interacción con el medio ambiente.

\section{Nivel Universitario}

Para los universitarios a través de un diálogo se logrará la identificación de las especies de las diferentes familias presentes en el Jardín Botánico con la finalidad de desarrollar la asignatura Botánica en condiciones naturales. El alumno caracterizará de 


\section{REVISTA REAMEC}

Revista da Rede Amazônica de Educação

em Ciências e Matemática

cada ejemplar: porte, tipo de tallo, hojas, clasificación por su limbo, forma, borde, nervios, disposición; flores clasificación por su perianto, órganos reproductores (androceo y gineceo); por sus frutos y comparará sus observaciones con las de otros ejemplares para el establecimiento de los caracteres diagnósticos e identificar Familias, Géneros y Especies (ROBLEDO et al., 2010).

Resultados de las visitas guiadas

En las visitas realizadas a los diferentes grupos, se comprobó la elevación de los conocimientos acerca de las plantas y la sensibilización hacia la temática, a partir de los resultados siguientes:

- Narraciones realizadas por los niños sobre la planta que más le impresionó.

- Dibujos relacionados con la presencia de especies de interés en las áreas didácticas y de colecciones.

- Solicitud de los niños y maestros de continuar asistiendo a actividades programadas por el jardín.

- Cartas de reconocimiento de los directores de centros estudiantiles avalando el trabajo realizado.

\subsection{Centros que han participado en las visitas guiadas}

\section{Escuelas Primarias}

Desde 1996 hasta el 2015 se han atendido grupos de estudiantes de la enseñanza primaria, con el objetivo de aportar conocimientos a los programas que tienen contenidos relacionados con el cuidado del medioambiente.

El total de visitas realizadas por los niños de las escuelas primarias al jardín es de 688 , los cuales aparecen en las firmas recogidas en el libro de visitas. No se han registrado las participaciones en actividades convocadas por el jardín realizadas en las escuelas u otras áreas naturales.

Dentro de las escuelas Primarias que han participado están: "Reynold García", "Seguidores de Camilo y Che”, "René Fraga Moreno", "Francisco Vega Alemán”, "Sí por Cuba", "José Luis Dubrocq", "Leonor Pérez”, "Mártires de Corinthia" (Figura 1). 


\section{REVISTA REAMEC}

Revista da Rede Amazônica de Educação

em Ciências e Matemática
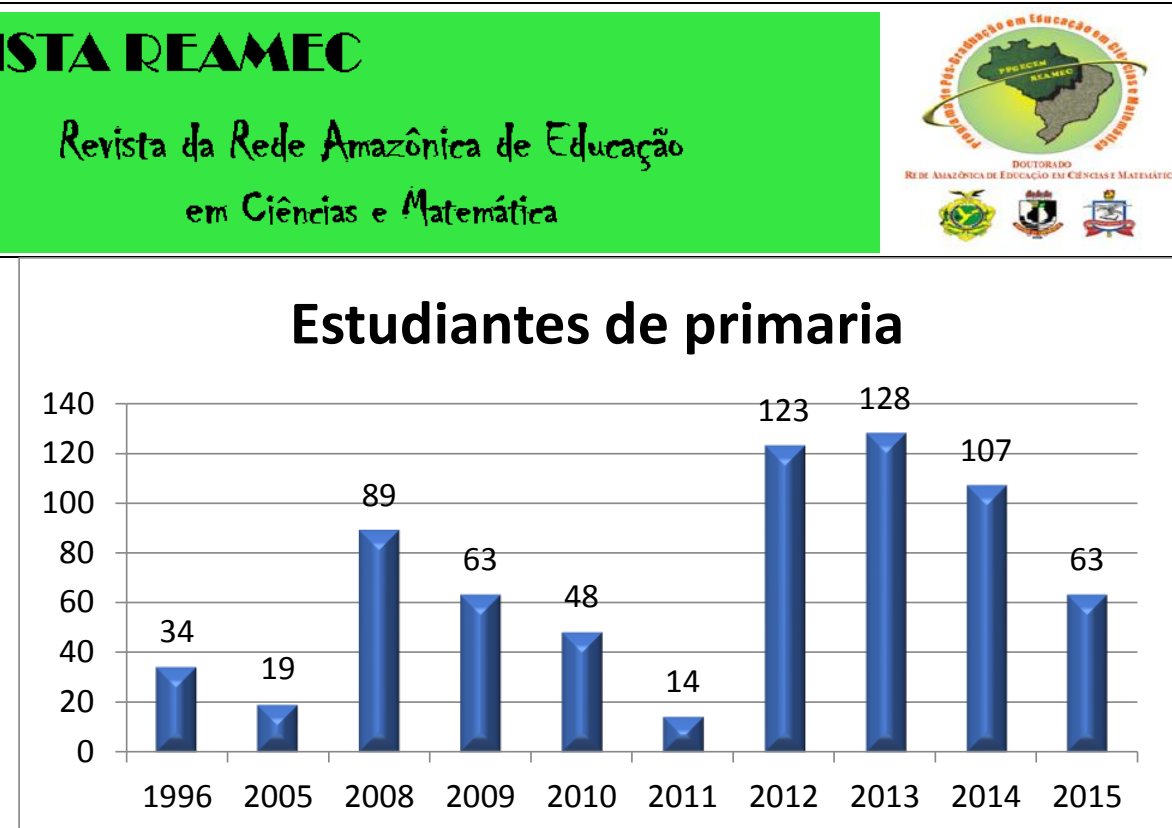

Figura 1: Cantidad de estudiantes de Primaria en visitas guiadas al Jardín Botánico de Matanzas.

En los años 2012 al 2014, se realizaron el mayor número de visitas con los estudiantes de nivel primario, teniéndose como promedio 16 niños por cada visita programada para la escuela (Figura 2 a y b).

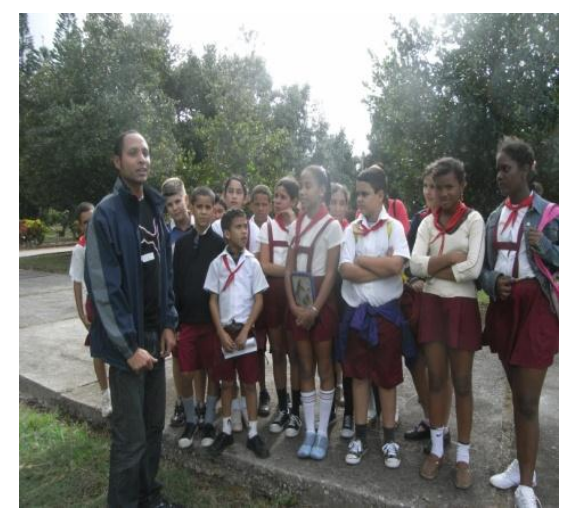

Figura 2 a: Visita guiada con niños de la escuela primaria "René Fraga Moreno".

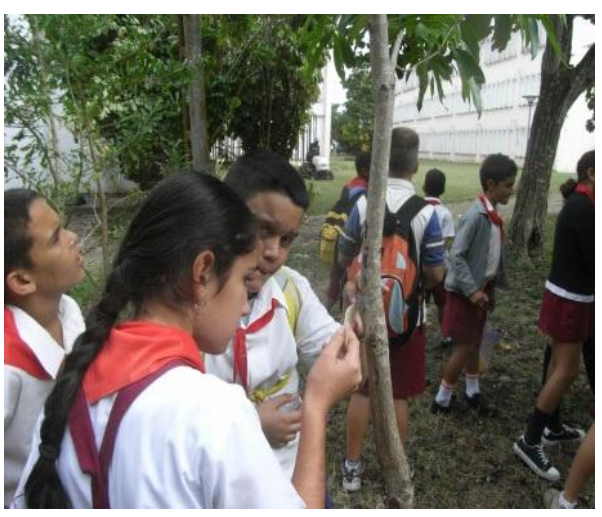

Figura 2 b: Observación de las características de órganos de las plantas del bosque martiano.

\section{Escuelas secundarias}

Desde 1996 hasta el 2014 los grupos de secundaria básica atendidos lograron recolectar conos de pinos, flores de diversas angiospermas y realizar actividades de protección hacia el medioambiente.

El total de visitas realizadas por los adolescentes de las escuelas al jardín, fue de 303. El registro de sus firmas se realizó solo para las actividades dentro del campus universitario y en sus centros se realizaron buzones del saber, encuentros de conocimientos, defensa de trabajos de los grupos científicos y de orientación vocacional, así como programas televisivos. 


\section{REVISTA REAMEC}

\section{Revista da Rede Amazônica de Educação em Ciências e Matemática}

Las escuelas secundarias incorporadas a las visitas guiadas fueron: "Antonio Berdayes”, "Héroes del Moncada”, "Protesta de Baraguá”, “Cándido González”, Escuela de Arte "Alfonso Pérez Isaac", todas del Municipio Matanzas. (Figura 3).

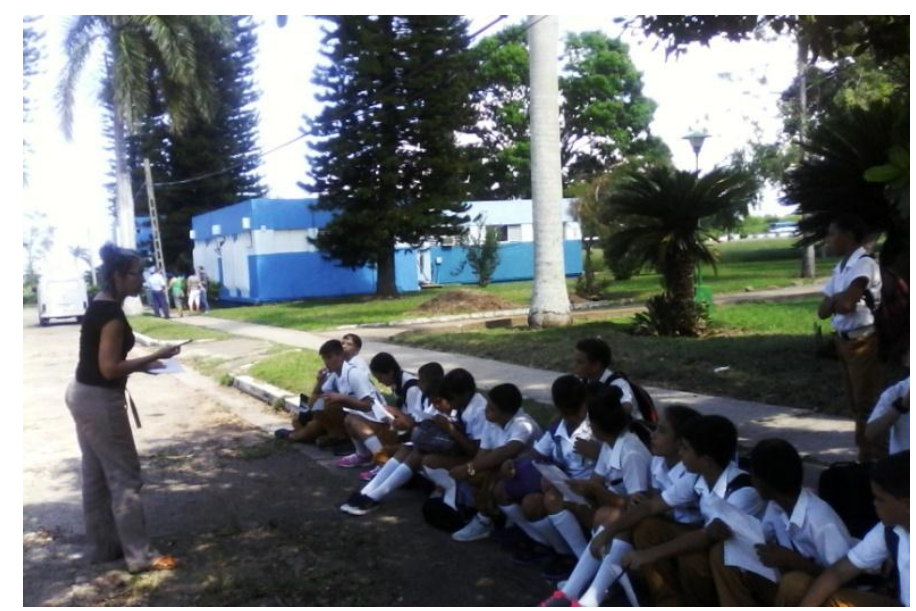

Figura 3: Estudiantes de la escuela secundaria "Cándido González", en visita al Jardín Botánico de Matanzas.

Los contenidos de Biología 1 impartidos en séptimo grado propiciaron el desarrollo de actividades prácticas en las áreas didácticas del jardín, donde diferenciaron las plantas gimnospermas de las angiospermas, grupos representados en las colecciones vivas.

\section{Estudiantes universitarios}

Los grupos de estudiantes de las diferentes carreras de la Universidad de Matanzas participan en las visitas guiadas y otras actividades ambientalistas en el Jardín Botánico, a partir de las estrategias curriculares de medioambiente de las facultades, carreras, departamentos y asignaturas.

Los estudiantes de la carrera de Agronomía por sus contenidos reciben clases prácticas para el desarrollo de habilidades relacionados con las asignaturas de Botánica, Silvicultura, Sanidad Vegetal, optativas Plantas Medicinales y Plantas Ornamentales. La asignatura Botánica explota las potencialidades del jardín botánico a través de la caracterización de los órganos vegetativos y reproductivos, identificación de los diferentes taxa y la ubicación taxonómica de especies de interés variado.

Durante el período de 1998 a 2015 se atendieron más de 600 estudiantes en las visitas guiadas, elevándose la cultura ambientalista, el cuidado del medio ambiente y el amor por la naturaleza (Figura 4). 


\section{REVISTA REAMEC}

Revista da Rede Amazônica de Educação

em Ciências e Matemática

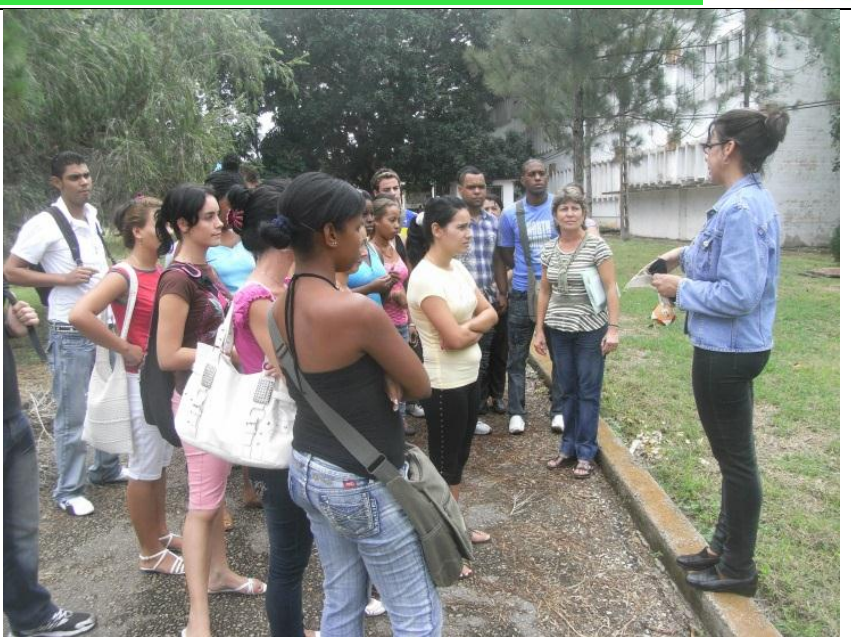

Figura 4: Estudiantes universitarios de la Carrera Ingeniería Química en visita guiada en el Jardín Botánico de Matanzas.

El resultado acerca de las visitas guiadas que se contabilizó en 688 estudiantes de primaria, 303 de secundaria básica y 600 universitarios, en los diferentes cursos ha sido un logro del jardín botánico de Matanzas, a partir de que la atención a las visitas era la responsabilidad de estudiantes del grupo científico del jardín. En los últimos años se elevaron las visitas en un $50 \%$ con la presencia de un especialista en educación ambiental potenciándose la atención hacia los estudiantes universitarios. (Figura 5).

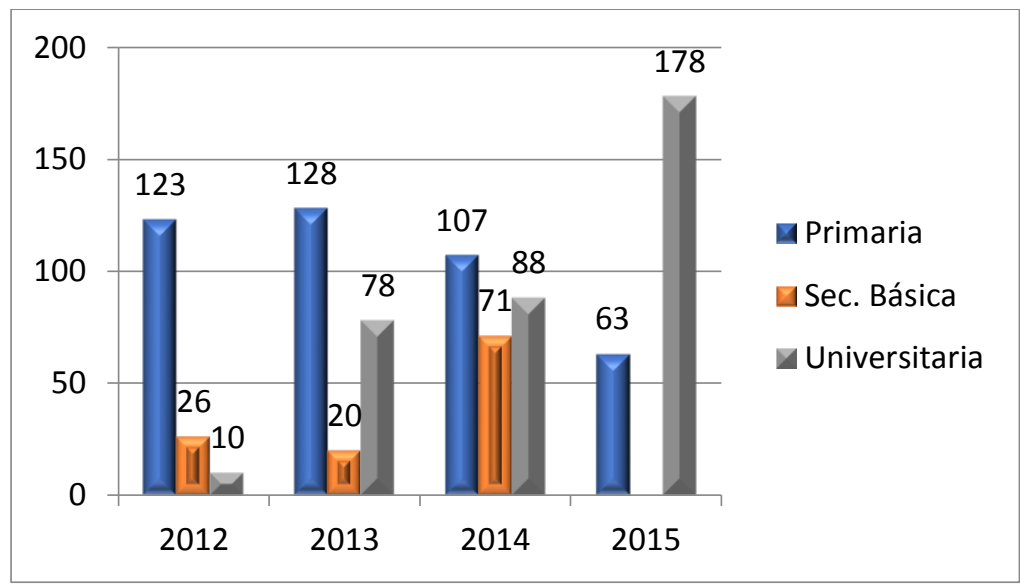

Figura 5: Cantidad total de estudiantes por niveles de enseñanza en visitas guiadas al Jardín Botánico de Matanzas en los períodos del 2012 al 2015.

Durante el desarrollo del jardín botánico se han realizado un total de 1792 visitas guiadas incluyendo además de los estudiantes de los diferentes niveles educacionales a: estudiantes de la Universidad del adulto Mayor, Grupo de Inspección Estatal, Miembros de la Asociación Nacional de Aficionados a la Botánica y Protección de la Naturaleza “Juan Tomás Roig”, Filial Matanzas; estudiantes del colegio “Gérald Godin” de Québec, Canadá, Círculos de Abuelos, Profesores Invitados de Estados unidos, Alemania y 


\section{REVISTA REAMEC}

Revista da Rede Amazônica de Educação

em Ciências e Matemática

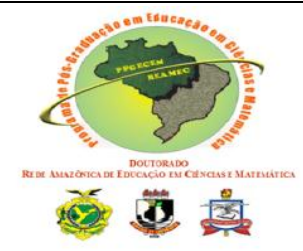

Canadá; especialistas de La Empresa provincial para la Protección de la Flora y la Fauna, equipo de Medicina Natural y Tradicional de la provincia de Matanzas, Estudiantes sanitarios del Hospital Militar "Mario Muñoz Monrroy" y Servicio Estatal Forestal, con el fin de intercambio, inspecciones, visitas, que avalan el trabajo de la educación ambiental en el Jardín Botánico de Matanzas.

\section{CONSIDERAÇÕES FINAIS}

El Jardín Botánico de Matanzas desarrolla dentro de sus líneas de trabajo, la educación ambiental con los estudiantes de los diversos niveles educacionales, así como otras instituciones y personalidades que visitan el jardín.

Las visitas guiadas son las formas principales para el desarrollo de las acciones medioambientalistas que se adecuan a las características de los diferentes grupos y elevan los conocimientos acerca de las plantas y la importancia de su protección.

Los resultados de las visitas guiadas avalan el trabajo de la educación ambiental en el Jardín Botánico de Matanzas, Cuba.

\section{REFERÊNCIAS}

ÁLVAREZ, A. El Sector Forestal Cubano y el Cambio Climático. La Habana. Proyectos. 2013.

BACCHETTA, G.; BUENO, A.; FENU, G.; JÍMENEZ-ALFARO, B.; MATTANA, E.; PIOTTO, B.; VIREVAIRE, M. Conservación ex situ de Plantas Silvestres. El Papel de los Jardines Botánicos. Principado de Asturias/ la Caixa. 2008.

GALLEGO, J. H. La educación ambiental como estrategia espiritual en la conservación de especies en vías de extinción de Colombia, en el Jardín Botánico de la Universidad de Caldas. Revista jardín Botánico Nacional. Vol. XXX-XXXI, 2010, p. 101-103.

GONZÁLEZ, A. Jardín Botánico de la Universidad de Matanzas, Potencialidades para el desarrollo de la Biología 1 de $7^{\mathrm{mo}}$ grado. Trabajo de Diploma en opción al título de Licenciado en Ciencias Biológicas. Instituto Superior Pedagógico "Juan Marinello". Matanzas. 2001.

GONZÁLEZ, A.; CAPITANGO, P.; ROBLEDO, L. Aulas Práticas de Botánica no Instituto Superior de Ciências de Educação do Huambo, Angola. Revista REAMEC, Cuiabá - MT, n.03, dezembro 2015.

GONZÁLEZ, A.; ROBLEDO, L.; ENRÍQUEZ, A. O papel do Jardim Botânico em desenvolvimento científico como um tributo à Conservação da Biodiversidade vegetal. 


\section{REVISTA REAMEC}

Revista da Rede Amazônica de Educação

em Ciências e Matemática

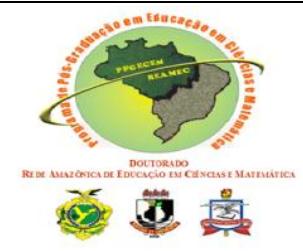

Experienciências do jardim Botânico de Matanzas, Cuba. Revista Ethos \& Episteme. v. 10, n 19. jan-jun 2014.

LINARES, E.; HERNÁNDEZ, C.; HERRERA, E. La Educación en Los Jardines Botánicos: Un Mundo de Ideas. Jardín Botánico de la Universidad Autónoma de México. UNAM. México. Publicación Especial n. 3, 1994.

RANGEL, L. M. Visitas guiadas y audiovisuales: una actividad constante y enriquecedora para el público que visita los Jardines Botánicos en La Educación en los Jardines Botánicos un Mundo de Ideas. Asociación Mexicana de Jardines Botánicos. México DF, v. 3, 1994, p. 50-59.

ROBLEDO, L.; ENRÍQUEZ, A.; GONZÁLEZ, A.; DOMECH, R.; DOMÍNGUEZ, B.; TERRY, M.; MESTRE I. La Educación Ambiental en el Jardín Botánico de Matanzas. CIUM, 2009.

ROBLEDO, L.; ENRÍQUEZ, A.; GONZÁLEZ, A.; CRUZ, R. El Jardín Botánico de Matanzas y la conservación de las especies amenazadas de la provincia. Revista Jardín Botánico Nacional. v. 30-31, 2009-2010, p. 73-83.

ROBLEDO, L.; ENRÍQUEZ, A.; GONZÁLEZ, A.; TERRY, M.; MESTRE, I. Perfeccionamiento de la asignatura de Botánica a partir de la contribución de la actividad laboral investigativa en el primer año de la carrera de Agronomía, Johannes Bisse, Camagüey, Cuba. Sello Editor educación Cubana. 2010.

ROBLEDO, L.; GONZÁLEZ, A.; ENRIQUE, A.; RODRÍGUEZ, Y. Potencialidades etnobotánicas de la colección viva del Jardín Botánico de Matanzas y Ia Educación Ambiental. Octubre de 2015.

Submetido em: 11 de março de 2018

Aprovado em: 13 de maio de 2018 